\title{
Die Bildung der Gefühle
}

\section{ZfE}

\author{
Ute Frevert · Christoph Wulf
}

Zusammenfassung: Das, was Friedrich Schiller „Bildung des Herzens“ und Wilhelm von Humboldt „Bildung des Gemüths“ nannten, gilt in der deutschen Tradition als wichtiges Element „vollständiger Menschenbildung“. Wie aber konnte eine solche Bildung aussehen? Wie ließen sich Neid und Gier, Zorn und Hass in der Gesellschaft minimieren? Wie weckte man Mitgefühl? Schon in der Antike, besonders aber seit dem 18. Jahrhunderts beschäftigten diese Fragen viele Pädagogen und Bildungstheoretiker. Der Streit, wie solche Bildungsziele erreicht werden können, ist heute kaum weniger heftig als damals. Angesichts der Rolle, die Gefühle in Politik, Wirtschaft und Medien, in Gender-Fragen sowie in der psychotherapeutischen Arbeit spielen, ist ihre Bedeutung für die menschliche Entwicklung offensichtlich. Dieses Sonderheft untersucht die Bildung der Gefühle in vier zentralen Bereichen: Kindheit und Familie, Peergroup und Schule, Medien und Bildung, Kultur und Lernen.

Schlüsselbegriffe: Herzensbildung $\cdot$ Bildung der Gefühle $\cdot$ Gefühl $\cdot$ Emotion

\section{Education of emotions}

\begin{abstract}
What Friedrich Schiller called "education of the heart" and Wilhelm von Humboldt called "education of a state of mind" is seen as an important element of comprehensive human development in the German education tradition. But what would such an education look like? How can jealousy, greed, anger and hate be minimized in society? How can empathy be awakened? Such questions have occupied educationalists and pedagogues since antiquity, and especially since the eighteenth century. The debate on how such educational goals could be reached is hardly less fiercely discussed today. In view of the role which feelings play in politics, industry and media, in gender questions and psychotherapeutic work, their importance for human development is plain to see. This special edition investigates the education and formation of emotions in four central areas: childhood and family, peer groups and school, media and education, culture and learning.
\end{abstract}

Keywords: Education of the heart $\cdot$ Education of emotions $\cdot$ Feelings $\cdot$ Emotion

(C) VS Verlag für Sozialwissenschaften 2012

Prof. Dr. U. Frevert

Max-Planck-Institut für Bildungsforschung, Lentzeallee 94,

14195 Berlin, Deutschland

E-Mail: sekfrevert@mpib-berlin.mpg.de

Prof. Dr. C. Wulf $(\bowtie)$

Freie Universität Berlin, Arnimallee 11, 14195 Berlin, Deutschland

E-Mail: chrwulf@zedat.fu-berlin.dew 
I.

Die deutsche Sprache kennt ein schönes Wort: Herzensbildung. Es ist so wenig in andere Sprachen übersetzbar wie der Begriff der Bildung überhaupt, in dem sich viele Bedeutungen mischen und der Prozesse des Entstehens und Entwickelns ebenso umfasst wie gezielte Anstrengungen der Selbstvervollkommnung und Erziehung durch andere.

Von Herzensbildung sprach man besonders gern in jener Epoche, die verklärend-pathetisch als „Goethezeit" firmiert. Ein idealistischer und idealisierender Ton schwang mit, wenn sich Dichter und Pädagogen über die „Bildung des Herzens“ (Schiller 1784) oder die „Bildung des Gemüths“ (von Humboldt 1982) ausließen. Nicht nur der Verstand bedurfte der Bildung und Aufklärung; es reichte nicht aus, bloß „richtigere Begriffe, geläuterte Grundsätze“ und das „Licht der Weisheit“ unter die Menschen zu bringen. Ebenso wichtig war es, „reinere Gefühle [...] durch alle Adern des Volks“ fließen zu lassen, „Menschlichkeit und Sanftmut in unser Herz“ zu senken (Schiller 1784, S.237, 244 f.; von Humboldt 1982, S. 190).

Das Herz galt damals als Sitz von Gefühl und Gemüt (Rolfus und Pfister 1863, S.394396 - „Herz, dessen Bildung“; Scheller 1780; Rüdiger 1990). Noch heute benutzen wir Ausdrücke und Zeichen, die sich dieser Topographie verschreiben: Wer liebt, verschenkt Ringe oder Schokolade in Herzform; wer Liebeskummer hat, dem wurde das Herz gebrochen, und es schmerzt ihn am und im Herzen. Wer glücklich ist, dem weitet sich das Herz, und wer trauert, dem krampft es sich.

Wie aber lässt sich ein Herz bilden? Sind Gefühle nicht angeboren und damit eigentlich unverfügbar? Haben wir nicht alle, wie viele Psychologen behaupten, ein Set sogenannter Basisgefühle, wie Angst und Freude, Hass und Trauer, Ekel und Wut? Und folgen jene Gefühle nicht einem natürlichen Rhythmus, einem biodynamischen Konzept, das sich den Menschen evolutionär vermittelt und eingeprägt hat? Was könnte unter solchen Umständen Bildung heißen und bewirken?

\section{II.}

Das sind Fragen, die schon in der Antike diskutiert wurden, die aber seit dem sogenannten pädagogischen Jahrhundert - dem achtzehnten - an Bedeutung und Sprengkraft gewannen. Wer den Menschen in Körper, Geist und Seele als bildungsfähiges und bildungsbedürftiges Wesen ansah und wer sich von seiner Bildung einen zivilisatorischen Fortschritt versprach, konnte an der Herzensbildung nicht achtlos vorbeigehen. Gebildet werden sollte nicht nur der Geist, der Verstand, also das, was Humboldt „Kenntnis der Kenntnis“ nannte. Gebildet werden sollten auch Gefühle und Empfindungen. Sie galten einerseits als naturgegeben und dem Menschen unmittelbar zugänglich. Andererseits schien es problematisch, sie im ungefilterten Naturzustand zu belassen. Sicher war jeder Mensch, wie die schottischen Moralphilosophen betonten, fähig zum Mitgefühl (sympathy). Aber ob diese Fähigkeit auch genutzt und gepflegt wurde, stand auf einem anderen Blatt. Bei manchen Menschen konnte sie verschüttet oder von konkurrierenden Gefühlen der Selbstliebe bedrängt sein. Hier tat Erziehung not, um das, was dem Menschen eignete, auch zum Vorschein kommen zu lassen und in die Praxis umzusetzen. 
Andere Gefühle - Neid und Gier, Zorn und Hass - waren hingegen vielleicht im Übermaß vorhanden und störten das gedeihliche Zusammenleben in einer bürgerlichen Gesellschaft. Wer sich von solchen negativen Gefühlen und Leidenschaften beherrschen ließ, war eine Gefahr für sich selber und für andere. Auch hier mussten Eltern und Erzieher intervenieren, um Exzesse zu verhindern und für wohltemperierte emotionale Haushalte zu sorgen. Das Ziel solcher Interventionen war der selbstgesteuerte Mensch, der sich beobachtete, kontrollierte, kultivierte und dessen Gefühlsvermögen den Prozess der Zivilisation unterstützte, anstatt ihn zu behindern.

Wie wichtig die Aufklärungspädagogik eine solche Bildung der Gefühle nahm, lässt sich an ihren praktischen Erziehungslehren deutlich ablesen. Basedows Elementarwerk von 1774 bspw. räumte den „Trieben“, „Begierden“, „Affekten“ und „Gemüthsbewegungen“ der Kinder einen zentralen Platz ein. Unterstützt von Daniel Chodowieckis Kupfertafeln, erfuhren Schüler, Lehrer und Eltern einen gründlichen Gefühlsunterricht: Sie lernten, was gute und schlechte, positive und negative, heftige und sanfte Gefühle waren, welche zu welcher Lebenssituation und Beziehung passten und welche unbedingt vermieden werden sollten. Furcht und Raserei, Ekel, Trauer und Verwunderung wurden hier ebenso in Szene gesetzt wie „feindselige Affekte“ oder die „Neigung zu Geiz, Wollust und Stolz“. Besonderes Gewicht erhielten „Selbstliebe und Menschenliebe“, Mitleid, Ehrliebe und Dankbarkeit. Der ,geordnete Vorrath aller nöthigen Erkenntniß zum Unterricht der Jugend" enthielt folglich ein ausgefeiltes Bildungs- und Bildprogramm menschlich-kindlicher Gefühle. ${ }^{1}$

Über die konkrete Praxis und Organisation jenes Programms aber gab es schon unter den Zeitgenossen Dissens. Manche Pädagogen legten großes Gewicht auf eine ästhetische Erziehung, die Gefühle des Schönen und Erhabenen in der jugendlichen Seele verankern sollte. Eine frühe Hinführung zu Musik, Lyrik und bildender Kunst schien dafür unverzichtbar. Andere warnten vor zu viel Schöngeistigem. Die Lektüre von Romanen galt ihnen als gefährlich, für beide Geschlechter: Bei Frauen konnte sie zu übersteigerter Einbildungskraft und unerfüllbaren Wunschphantasien führen, die das wahre Leben nur enttäuschen würde. Junge Männer vergäßen über den Romanen ihre eigentliche Bestimmung in der Welt und bildeten zartbesaitete Gemüter aus, die mit ihren handfesten Pflichten in Politik, Wirtschaft und Gesellschaft schwer vereinbar seien. Zwar stand Empfindsamkeit in der zweiten Hälfte des 18. Jahrhunderts hoch im Kurs, und nicht nur Gotthold Ephraim Lessing hielt den mitleidigen Menschen für den besten. Dennoch durfte man des Guten nicht zu viel tun. Wem die Tränen allzu locker saßen, wer daraus geradezu einen Kult des authentischen Gefühls machte, der gab sich, wie Immanuel Kant kritisierte, der „Empfindeley“ hin. Gefragt war nicht bloß ein mitleidiges Herz, sondern auch Mut und Energie, das empfundene Mitgefühl in die Tat umzusetzen und aktiv zu helfen, wo es not tat.

Dass Gefühlsbildung ihren Ort auch, wenn auch nicht allein in der Schule fand, war unter Pädagogen unumstritten. Ebenso einig war man sich darin, dass sie stets mit Verstandesbildung einhergehen müsse. Wer bei Kindern und jungen Leuten nur Empfindungen und Empfindsamkeit kultiviere, erziehe Schwärmer und Enthusiasten, warnte 1780 der Gymnasialrektor Immanuel Johann Gerhard Scheller. Der Verstand dürfe nicht schlafen, sondern müsse das Gefühl und die Phantasie kenntnisreich und urteilsstark an die Hand nehmen. Joachim Heinrich Campe, Hauslehrer der Humboldt-Söhne und einfluss- 
reicher Schulreformer, verwendete das Bild vom Körper als Schiff, dem die Vernunft das Steuer führte und die Empfindsamkeit die Segel setzte (zit. in Heinze 2008, S. 151 f.).

Schulische Lehrpläne enthielten denn auch gemeinhin drei Schwerpunkte: Körper-, Geistes- und Herzensbildung. Seine Schüler, versprach der Leiter einer 1801 neugegründeten Berliner „Erziehungsanstalt für Söhne von sechs bis vierzehn Jahren“, würden dort zu ,moralisch guten Menschen“ erzogen, ,an Verstand und Herzen gebildet“, an „Reinlichkeit, Ordnung, Thätigkeit, Bescheidenheit und Gefälligkeit" gewöhnt und mit Liebe für ,,ihr Vaterland und die Verfassung desselben“ erfüllt. „Herzensbildung“ umfasste hier die „sanfte, liebreiche Lenkung der jugendlichen Triebe“, „Abmahnung von Fehltritten“ und „Vorstellung der natürlichen Folgen der Tugend und des Lasters durch Beispiele aus dem Menschenleben“ (Krüger 1801, S. 1-8).

\section{III.}

Wie „sanft“ und „liebreich“ Schulen im 19. Jahrhundert die Gefühle und „Triebe“ ihrer Zöglinge tatsächlich lenkten, steht auf einem anderen Blatt. In den Volksschulen beschränkte sich Herzensbildung im Wesentlichen darauf, den Jungen und Mädchen Pflichtbewusstsein und Gehorsam gegen die kirchliche und staatliche Obrigkeit beizubringen. Gymnasien warteten zwar mit einem differenzierteren moralischen und ästhetischen Curriculum auf. Doch nicht nur Harry Graf Kessler erlebte seine Hamburger Schulzeit in den 1880er Jahren als „Abrichtung“: „Wir sollten eigentlich gar nicht Griechisch oder Latein lernen, sondern Arbeiten. Arbeiten um seiner selbst willen; man wollte uns abrichten zu Arbeitstieren. Vom Ideal des humanen, die ganze Menschheit und ihre Kultur in Kopf und Herz tragenden Menschen, das die Goethezeit entflammt hatte, war nur der ungeheure Fleiß übriggeblieben, der nötig war, um den unermeßlichen Stoff aufzunehmen.“ Statt die „Seele sowohl nach ihrer Geistes- wie auch nach ihrer Gefühlsseite" aufzuschließen, vermittelte die Schule nurmehr Fertigkeiten und Einstellungen, die, wie Kessler schalt, „,en Herren der neuen Zeit die für die Mechanisierung der Wirtschaft benötigten unermüdlichen und selbstzufriedenen Sklaven“ lieferten (Kessler 1988, S. $128,131 \mathrm{f}$.).

Wenig Herz und Humanität ließ der gymnasiale Bildungskanon auch dort erkennen, wo es um soziale Fragen ging. Für die Sorgen, Nöte und Sehnsüchte unterbürgerlicher Schichten hatte man kein Sensorium. Je mehr sich zudem nationale Belange und Orientierungen in den Vordergrund schoben, desto blasser wurde auch die kosmopolitische Botschaft der Weimarer Dioskuren. Schillers emphatische Forderung und Prognose einer weltumspannenden Brüderlichkeit rückte in weite Ferne. Obwohl die Welt mittels kolonialer Großprojekte spürbar zusammenwuchs, war der imperiale Habitus nicht von Solidarität und Geschwisterliebe geprägt, sondern von sozialem, zunehmend rassisch überformten Überlegenheitsdünkel. Wer den eigenen zivilisatorischen Standards nicht entsprach, erntete Herablassung, Verachtung und, im Extremfall, Vernichtung.

Wie schlecht es um die allgemeine Herzensbildung bestellt war, zeigte sich nicht nur während des wilhelminischen Zweiten Reichs, das sich bei der aggressiven Identifizierung innerer und äußerer Feinde schwer überbieten ließ. Es zeigte sich noch viel radikaler und rabiater in den Jahren des nationalsozialistischen Dritten Reichs, das Feindschaft 
nicht allein politisch, sondern auch und v. a. rassisch begründete. Mitleid, hieß es 1939 in Meyers Lexikon (1939, Bd. 7, Sp. 1455), gelte lediglich für „Gemeinschaftsgenossen“; nur mit denjenigen, die zur Volksgemeinschaft gehörten, könne man miterleben und mitfühlen, und nur ihnen werde „tätige, leidlindernde oder leidbehebende Hilfe“ zuteil. Das Leid derer, die sich aus dieser Gemeinschaft ausgeschlossen sahen, zählte nicht. Wer ihnen Mitgefühl bekundete oder gar half, machte sich verdächtig und riskierte scharfe Sanktionen.

Gerade diese Erfahrung, so steht zu vermuten, bewog manche Zeitgenossen nach 1945, Gefühlsbildung als schulisches Erziehungsziel ausdrücklich zu betonen. Der Vorschlag des sozialdemokratischen Ministerpräsidenten Wilhelm Hoegner, dies in der neuen Verfassung des Freistaates Bayern zu verankern, wurde von der Verfassungsgebenden Landesversammlung einstimmig angenommen. Bis heute erlegt Paragraph 131 bayerischen Schulen die Aufgabe auf, „nicht nur Wissen und Können [zu] vermitteln, sondern auch Herz und Charakter [zu] bilden“. „Oberste Bildungsziele sind Ehrfurcht vor Gott, Achtung vor religiöser Überzeugung und vor der Würde des Menschen, Selbstbeherrschung, Verantwortungsgefühl und Verantwortungsfreudigkeit, Hilfsbereitschaft, Aufgeschlossenheit für alles Wahre, Gute und Schöne“. Außerdem seien Schüler ,,in der Liebe zur bayerischen Heimat und zum deutschen Volk und im Sinne der Völkerversöhnung zu erziehen". 2

Der Streit der Pädagogen, ob und wie solche Bildungsziele erreicht werden können, tobt heute nicht weniger heftig als um 1800. Manche lehnen sie als arrogante und naive Zumutung ab, andere bringen ein „Schatzbuch der Herzensbildung“ heraus und übersetzen letztere in die managementtaugliche Sprache der „emotionalen Intelligenz“ (Liebertz 2004; kritisch: März 1993, S. 321). Während viele Politiker dabei vornehmlich an Werteerziehung denken, experimentieren neuerdings immer mehr Schulen mit Empathietraining - die seit langem konkreteste und praktischste Umsetzung der alten Idee, dass ,allgemeine Menschenbildung“ nicht nur Körper und Geist, sondern auch das Herz und seine Gefühle umfasst.

Dem Ziel einer ,allgemeinen Menschenbildung“ ist auch die UNESCO-Empfehlung „Learning - The Treasure within“ verpflichtet, die unter Federführung von Jacques Delors für das 21. Jahrhundert erarbeitet wurde. Sie geht von einem Erziehungs- und Bildungsbegriff aus, der nicht nur den Erwerb von Wissen umfasst, sondern auf eine allgemeine Bildung des Menschen (human development) zielt. Diese soll in der globalisierten Welt vier Dimensionen umfassen: learning to know, learning to do, learning to live together/ learning to live with others, learning to be. ${ }^{3}$ Wenn die Entwicklung von Neugier in Bezug auf Wissen, die Herstellung von Dingen und das Realisieren von Handlungen, das Zusammenleben mit anderen und die Akzeptanz der individuellen Existenz Ziele menschlicher Entwicklung sind, spielen Gefühle dabei eine zentrale Rolle. Deshalb ist es merkwürdig, wie wenig die Bildung der Gefühle in den letzten Jahrzehnten in der Erziehungswissenschaft zum Thema geworden ist. (vgl. erste Ansätze dazu: Dörr und Göppel 2003; Klika und Schubert 2004;Wulf und Prenzel 2011; Nussbaum 2001.) 
IV.

Jene Bildung findet in allen gesellschaftlichen Bereichen statt, in denen Menschen agieren und interagieren (Friedelmeier und Holodynski 1999). Gefühle entstehen in sozialen und kulturellen Relationen; der Umgang mit ihnen wird gelernt. In mimetischen Prozessen beziehen sich Kinder und Jugendliche auf die Gefühle von Menschen, die ihnen wichtig sind. Sie nehmen wahr, wie diese Menschen welche Gefühle in welchen Situationen empfinden, wie sie Emotionen körperlich erfahren, inszenieren und aufführen, wie sie sie darstellen, sprachlich fassen und reflektieren. In solchen alltäglichen Praktiken überlagern sich unterschiedliche, manchmal auch widersprüchliche Gefühle, sodass häufig keine eindeutigen, sondern eher „gemischte“ Gefühle entstehen. Bei ihrer Genese und Ausprägung wirken kollektive und individuelle Elemente zusammen. Viele dieser mit dem praktischen Wissen der Menschen verbundenen Gefühle gelangen nicht ins Bewusstsein, sondern bleiben ,halbbewusst“ oder gar unbewusst. Die Erforschung dieser Prozesse steht noch am Anfang und stellt eine „Ethnographie der Emotionen“ vor schwierige Aufgaben.

Die Bildung der Gefühle findet nicht nur in den klassischen Institutionen menschlicher Erziehung und Bildung statt. Auch außerhalb dieser Institutionen werden Gefühle geformt, geprägt, verhandelt und in Frage gestellt (vgl. Böhme 2010). Einige gesellschaftliche Felder und Prozesse, die auch in den Beiträgen dieses Sonderhefts eine wichtige Rolle spielen, seien hier thesenartig skizziert:

Kommerzialisierung und Politisierung; Am Arbeitsplatz kommt Gefühlen und dem Umgang mit ihnen erhebliche Bedeutung zu. Häufig als „emotionale Intelligenz“ und „emotionale Kompetenz“ bezeichnet, sind sie bzw. ist der Umgang mit ihnen zentrale Voraussetzung für gelingende Kooperation in der Wirtschaft. Auch und gerade beim Marketing spielt das Kalkül mit den Emotionen der Konsumenten eine zentrale Rolle. In den kapitalistisch organisierten Gesellschaften durchdringt die Kommerzialisierung alle Bereiche menschlicher Beziehungen und Emotionalität (Martin et al. 2003; Gobé 2001). Auch aus der politischen Kommunikation sind Gefühle nicht wegzudenken: Politiker spielen mit den Ängsten und Hoffnungen der Menschen und manipulieren sie, um ihre politischen Ziele zu realisieren (Furedi 2005).

Gender und die Modellierung von Emotionen; Bereits in der Kindheit zeigt sich, dass Emotionen nicht gender-neutral sind. Wie sollen sich junge Mädchen und junge Frauen, wie Jungen und junge Männer fühlen, wie mit ihren Gefühlen umgehen? Gibt es genderspezifische Formen des Fühlens? Wie verbinden Jungen und junge Männer und Mädchen und junge Frauen Gefühle, die ihnen gemeinsam sind, mit denen, durch die sie sich voneinander unterscheiden? Gab es früher leicht identifizierbare Gender-Schemata in den Gefühlen von Männern und Frauen, scheinen sie heute ihre Eindeutigkeit und Trennschärfe weitgehend verloren zu haben (Butler 1990; Vandekerckhove et al. 2008).

Neue Medien; Besonders auffällig ist die Inszenierung und Aufführung von Gefühlen in den neuen Medien, in Talkshows, im Reality TV oder in Sendungen, in denen Millionen Zuschauer einzelne Personen in Situationen der Enttäuschung, Kränkung, Eifersucht usw. beobachten (Hill 2005). Offenbar erleben viele Menschen dadurch, dass sie ihre Gefühle öffentlich und vor Publikum darstellen, eine Intensivierung dieser Gefühle. Die anonyme Inszenierung von Gefühlen in Internetforen wie Facebook, Second Life, You 
Tube oder Twitter scheint ähnlich zu wirken: Sie hilft Menschen, Gefühle zu empfinden, die sie nicht haben, jedoch gerne hätten (Ben-Ze'ev 2004). Die weltweite mediale Verbreitung von Katastrophen wie Erdbeben, Tsunami-Wellen und Wirbelstürmen ist von der Zurschaustellung intensiver Gefühle des Leidens und Schmerzes begleitet, die bei den Zuschauern schaurige Lustgefühle wecken.

Psychotherapeutische Arbeit mit Gefühlen; Immer dann, wenn die Bildung der Gefühle nicht oder nur unzureichend gelungen ist, wird die psychotherapeutische Arbeit wichtig. Ihre Ausweitung und Professionalisierung reflektiert einen Problemdruck, der mit der Modernisierung der Gesellschaft, der Auflösung traditioneller Familienbindungen, der zunehmenden Individualisierung und den damit verbundenen emotionalen Verunsicherungen zusammenhängt. Viele Menschen sind nicht mehr in der Lage, mit ihren Gefühlen zurechtzukommen, und benötigen Hilfen. Die Sorge für sich und für das emotionale Wohlbefinden wird in modernen Gesellschaften zu einer wichtigen Bedingung für die Qualität individuellen und gemeinschaftlichen Lebens (vgl. Greenberg und Paivio 1997).

In diesen für die Bildung der Gefühle zentralen gesellschaftlichen Feldern lassen sich Gefühle als soziokulturelle Praktiken begreifen, die auf andere Menschen bezogen sind und Wirkungen auf sie haben. Wie sich diese Praktiken vollziehen, ist von den jeweiligen kulturellen Werten, Normen, Sprach- und Handlungsspielen (Wittgenstein) abhängig. Besondere Aufmerksamkeit verdienen mimetische Prozesse, in denen sich Kinder und Jugendliche auf zugleich rezeptive und aktive Weise dem Verhalten, Handeln und Fühlen anderer Menschen ,,anzuähneln“ versuchen. Mit ihrer Imagination nehmen sie gleichsam einen „Abdruck“ der Handlungen und emotionalen Äußerungen anderer vor und integrieren ihn in ihre mentale Welt (vgl. Tomasello 2006; Gebauer und Wulf 1998). Bei der Erforschung dieser mimetischen Prozesse kommt es darauf an, Gefühle nicht zu isolieren, $\mathrm{zu}$ verdinglichen, zu objektivieren. Gefühle sind keine Substanzen; sie sind mit vielen anderen Merkmalen des Menschen verbunden. Ohne Körper, Bewusstsein, Imagination und Sprache gäbe es keine Gefühle In vielen Fällen tragen Sprache und Imagination erst dazu bei, dass Gefühle entwickelt, empfunden und unterschieden werden können.

\section{V.}

Das Sonderheft ist in vier Abschnitte gegliedert, deren erster sich der Emotionsentwicklung in der Kindheit widmet. Dabei werden zunächst Geschwisterbeziehungen in der Frühen Neuzeit untersucht, vornehmlich unter Berücksichtigung religiöser Texte und der in ihnen enthaltenen Normen (Gestrich). Daran schließt sich eine dichte ethnografische Untersuchung zeitgenössischer Kinderkrippen an, die darlegt, wie wichtig die Spiegelung und Modulierung von Gefühlen in sozialen Situationen für die emotionale Bildung und Differenzierung ist. Mikroanalytisch wird herausgearbeitet, wie Gefühle zwischen Kindern in konkreten Interaktionen artikuliert und modifiziert werden und welche Herausforderungen dies für professionelle Erzieherinnen darstellt (Stenger). Bereits von Geburt an werden Emotionen in einem sozialen Miteinander gelernt. Die soziale Gemeinschaft ist eine Bedingung dafür, Empfindungen zu identifizieren. In diesen frühen Lernprozessen führt die gemeinsame Aufmerksamkeit von Kind und Bezugsperson auf einen Gegenstand zu einer Semantisierung der Gefühle. Die Verbindung von Emotion und Sprache 
schafft die Voraussetzungen dafür, sich und andere zu verstehen (Engelen). Zuwendung und Liebe spielen dabei eine wichtige Rolle. Aufgrund der verbreiteten Trennung von Vernunft und Gefühl, Kognition und Emotion ist nicht genügend gesehen worden, wie wichtig Liebe für die Sorge, das (Miteinander-) Handeln und die Reflexion dieses Handelns in pädagogischen Zusammenhängen ist (Seichter).

Der zweite Abschnitt stellt die Emotionsbildung in Jugendgruppen und in der Schule in den Mittelpunkt. Der erste Beitrag beleuchtet die Gefühlswelt in jugendbewegten Jungmännerbünden des frühen 20. Jahrhunderts. Er rekonstruiert erfahrungsgeschichtlich, wie die Alterskohorte junger Frontsoldaten und Kriegskinder des Ersten Weltkriegs von solchen Gefühlswelten geprägt wurde (Reulecke). Es folgt eine ethnographische Untersuchung, wie Mädchen in einer zeitgenössischen Peergroup ihre Gefühle inszenieren und aufführen, v. a. im Verhältnis zum Selbst, zu anderen und zur Welt (Tervooren). Der nächste Beitrag analysiert an empirischen Beispielen aus der heutigen Unterrichtspraxis den sozialen und performativen Charakter von Gesten und arbeitet den Zusammenhang zwischen Gesten und Emotionen heraus (Kellermann).

Im dritten Abschnitt wird in exemplarischer Absicht gezeigt, wie Emotionen in oder im Zusammenhang mit unterschiedlichen Medien dargestellt und modelliert werden. Der erste Beitrag zeigt, wie wichtig der literarische Umgang mit Angst für die allgemeine Menschenbildung ist. Er erinnert Leser und Leserinnen an ihre Vergänglichkeit, fordert sie zur Sorge um ihr Seelenheil auf und bringt sie dazu, ihre Gefühle zu kontrollieren und moralisch zu läutern. Die literarische Thematisierung soll den Leser mit den Grenzen menschlicher Planung und Macht konfrontieren (Watanabe-O'Kelly). Wie Angst kann die mit Verlust und Tod verbundene Trauer zu vielschichtigen theoretischen, praktischen und ästhetischen Veränderungen im Verhältnis zur Welt, im Verständnis anderer Menschen und im Selbstverständnis führen. Trauer entbindet Menschen, führt zu Fremdheitserfahrungen und zur Wahrnehmung menschlicher Zerbrechlichkeit (Böhner/Zirfas). Wie eng Gefühle und musikalisches Erleben miteinander vertaktet sind, ist Thema des nächsten Beitrags. Er untersucht die ästhetische, erotische und kommunikative Funktion von Emotionen anhand des Opernpublikums von Berlin, Wien und London im 19. Jahrhundert (Müller). Daran schließt sich eine Studie über virtuelle Umgebungen an, deren soziale und subkulturelle Räume zu Orten des Ausdrucks und der Artikulation, der Inszenierung und Darstellung von Emotionen werden. Dabei spielen Avatare eine wichtige Rolle, mit deren Hilfe komplexe Prozesse emotionalen Lernens organisiert werden. In diesen Prozessen spielen die szenische Situiertheit, die visuelle Artikulation, die hybriden Handlungsstrukturen und die hybride Präsenz eine wichtige Rolle (Jörissen).

Der vierte Abschnitt macht deutlich, welche Wirkungen Religion, Nation und Kultur auf die Bildung der Gefühle haben. Im ersten Beitrag geht es um die Gotteserfahrung im Protestantismus und die Entwicklung einer spezifischen Innerlichkeit. Ausgefeilte Gefühlspraktiken helfen, die Aufmerksamkeit nach innen zu richten, wo Liebe, Glaube und Hoffnung, eng miteinander verbunden, eine innige Beziehung zu Gott stiften. Innerlichkeit wird entsprechend hoch bewertet, im Kontrast zum äußeren, an seinen Leib gebundenen Menschen (Scheer). Wie Religion ist auch Nation ohne ausgeprägte Emotionen nicht denkbar und erlebbar. Dass Liebe und Hass zur Bildung von Nationen beitragen, ist in der Nationsforschung unbestritten. Wenig Beachtung aber finden die Wirkungen nationaler Gefühle im Privaten und in der Öffentlichkeit und wie diese beiden sozialen Felder 
miteinander verbunden sind. An zwei Briefserien aus dem Ersten Weltkrieg wird gezeigt, wie sich Autoren im privaten Bereich gegen eine „national-emotionale Hochrüstung“ sperren (Langewiesche). Der abschließende ethnographische Beitrag nimmt die zentrale Bedeutung von Kultur und kulturellen Unterschieden bei der Bildung von Gefühlen in den Blick. In kulturvergleichender Absicht werden die Erziehungs- und Sozialisationspraktiken unter den Bara in Madagaskar und den Tao auf der taiwanesischen Insel Lanyu untersucht. Dabei spielen Furcht und Scham, aber auch Ärger und Trauer eine wichtige Rolle (Funk/Röttger-Rössler/Scheidecker).

Die Mehrzahl der Beiträge geht auf eine im Dezember 2010 gemeinsam veranstaltete Tagung zurück.

Berlin, im Juni 2012 Ute Frevert und Christoph Wulf

\section{Anmerkungen}

1 Basedow 1774; ähnlich Joachim Heinrich Campe, dessen Kleine Seelenlehre für Kinder 1784 erstmals erschien und bis 184412 Auflagen erlebte.

2 Verfassung des Freistaates Bayern vom 2.12. 1946, Dritter Hauptteil, 2. Abschnitt: Bildung und Schule. Stenographische Berichte über die Verhandlungen des Verfassungs-Ausschusses der Bayerischen Verfassungsgebenden Landesversammlung, Bd. 1. - München 1946, S. 257 f.

3 Learning - The Treasure within, ed. UNESCO. - Paris 1996; deutsche Fassung: Lernfähigkeit: Unser verborgener Reichtum. UNESCO-Bericht zur Bildung für das 21. Jahrhundert, hg. von der Deutschen UNESCO-Kommission. - Neuwied 1997.

\section{Literatur}

Basedow, J. B. (1774). Elementarwerk (4 Bde). Dessau: Selbstverlag.

Ben-Ze'ev, A. (2004). Love online: Emotions on the internet. Cambridge: Cambridge University Press.

Böhme, H. (2010). Gefühl. In Ch. Wulf (Hrsg.), Der Mensch und seine Kultur. Hundert Beiträge zur Geschichte, Gegenwart und Zukunft menschlichen Lebens (S. 525-548). Köln: Anaconda.

Butler J. (1990). Performative acts and gender constitution. An essay in phenomenology and feminist theory. In S.-E. Case (Hrsg.), Performing feminism. Feminist critical theory and theatre (S. 270-282). Baltimore: John Hopkins University Press.

Dörr, M., \& Göppel, R. (Hrsg.). (2003). Bildung der Gefühle. Gießen: Psychosozial.

Friedelmeier, W. H., \& Holodynski, M. (1999). Emotionale Entwicklung. Funktion, Regulation und soziokultureller Kontext. Heidelberg: Spektrum Akad.

Furedi, F. (2005). Politics of fear. Beyond left and right. London: Continuum.

Gebauer, G., \& Wulf, Ch. (1998). Spiel, Ritual, Geste. Mimetisches Handeln in der sozialen Welt. Reinbek: Rowohlt

Gobé, M. (2001). Emotional branding. The new paradigm of connecting brands to people. New York: Allworth.

Greenberg, L., \& Paivio, S. C. (1997). Working with emotions in psychotherapy. New York: Guilford Press. 
Heinze, K. (2008). Zwischen Wissenschaft und Profession. Das Wissen über den Begriff ,, Verbesserung "im Diskurs der pädagogischen Fachlexikographie vom Ende des 18. bis zur Mitte des 19. Jahrhunderts. Opladen: Budrich University Press.

Hill, A. (2005). Reality TV: Audiances and popular factual entertainment. London: Routledge.

von Humboldt, W. (1982). Schriften zur Politik und zum Bildungswesen (3. Aufl). Darmstadt: Wiss. Buchges.

Kessler, H. G. (1988). Gesichter und Zeiten. Erinnerungen. Frankfurt a. M.: Fischer-Taschenbuch. Klika, D., \& Schubert, V. (Hrsg.). (2004). Bildung und Gefühl. Baltmannsweiler: Schneider.

Krüger, W. (1801). Kurze Darstellung der Grundverfassung meiner in Berlin bestehenden Erziehungsanstalt für Söhne von sechs bis vierzehn Jahren. In Jahrbücher der preußischen Monarchie unter der Regierung Friedrich Wilhelms des Dritten (Bd. 2). Berlin: Unger.

Liebertz, Ch. (2004). Das Schatzbuch der Herzensbildung. Grundlagen, Methoden und Spiele zur emotionalen Intelligenz. München: Don Bosco.

Martin, B., Roach, A., \& Sharyn-Zadoroznyj, M. (2003). Editor's introduction to the special issue „Commercializing Emotions“. Journal of Sociology, 39.

März, F. (1993). Macht oder Ohnmacht des Erziehers. Bad Heilbrunn: Klinkhardt.

Meyers L. (1939). 8. Aufl., Bd. 7. Leipzig: Bibliographisches Institut.

Nussbaum, M. C. (2001). Upheavels of thought. The intelligence of emotions. Cambridge: Cambridge University Press.

von Rolfus, H., \& Pfister, A. (Hrsg.). (1863). Real-Encyclopädie des Erziehungs- und Unterrichtswesens nach katholischen Principien (Bd. 2). Mainz: Florian Kupferberg.

Rüdiger, H. (1990). Die Metapher vom Herzen in der Literatur. In von W. R. Berger \& E. Koppen (Hrsg.), Goethe und Europa (S. 117-159). Berlin: de Gruyter.

Scheller, I. J. G. (1780). Kurzgefaßte Gedanken über die Bildung des Herzens, ob sie ohne Bildung des Verstandes möglich sey, und zugleich über Empfindsamkeit, Unempfindsamkeit und Enthusiasmus. Breslau.

Schiller, F. (1784). Die Schaubühne als eine moralische Anstalt betrachtet. In Schillers Werke (Bd. 1, S.237-247). Berlin: Aufbau (1967).

Tomasello, M. (2006). Die kulturelle Entwicklung des menschlichen Denkens. Zur Evolution der Kognition. Frankfurt a. M.: Suhrkamp.

Vandekerckhove, M., von Scheve, Ch., Ismer, S., Jung, S., \& Kronast, S. (Hrsg.). (2008). Regulating Emotions. Culture, Social Necessity, and Biological Inheritance. Malden: Blackwell.

von Wulf, Ch., \& Prenzel, M. (Hrsg.). (2011). Themenschwerpunkt Emotion. Zeitschrift für Erziehungswissenschaft, 14(2).

Ute Frevert, Dr. phil., ist Direktorin des Max-Planck-Instituts für Bildungsforschung in Berlin und Honorarprofessorin an der FU Berlin. Sie ist Historikerin und arbeitet über die Geschichte der Gefühle in der Moderne.

Christoph Wulf, Dr. phil., ist Professor für Anthropologie und Erziehung, Mitglied des Interdisziplinären Zentrums für Historische Anthropologie, des Exzellenzclusters „Languages of Emotion“ und des Graduiertenkollegs „InterArts Studies“ an der Freien Universität Berlin. Seine Arbeitsschwerpunkte liegen in der historisch kulturellen Anthropologie und in der Pädagogischen Anthropologie. Er ist Vizepräsident der Deutschen UNESCO-Kommission. 